\title{
Puerarin pretreatment attenuates cardiomyocyte apoptosis induced by coronary microembolization in rats by activating the PI3K/Akt/GSK-3 $\beta$ signaling pathway
}

\author{
Zhi-Qing Chen ${ }^{1}$, You Zhou' ${ }^{1}$ Jun-Wen Huang ${ }^{1}$, Feng Chen ${ }^{2}$, Jing Zheng ${ }^{1}$, Hao-Liang Li ${ }^{1}$, Tao Li ${ }^{1}$, and Lang Li ${ }^{1 \text { ** }}$ \\ 'Department of Cardiology, The First Affiliated Hospital of Guangxi Medical University \& Guangxi Key Laboratory Base of Precision Medicine in Cardio-cerebro- \\ vascular Diseases Control and Prevention \& Guangxi Clinical Research Center for Cardio-cerebrovascular Diseases, ${ }^{2}$ Department of Emergency, The First Affili- \\ ated Hospital of Guangxi Medical University, Nanning, Guangxi 530021, China
}

\section{ARTICLE INFO \\ Received September 4, 2020 \\ Revised October 15, 2020 \\ Accepted October 26, 2020 \\ *Correspondence \\ Lang Li \\ E-mail: drlilang99@126.com}

\section{Key Words}

Apoptosis

Coronary microembolization

Myocardial injury

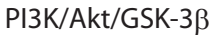

Puerarin

\begin{abstract}
Coronary microembolization (CME) is associated with cardiomyocyte apoptosis and cardiac dysfunction. Puerarin confers protection against multiple cardiovascular diseases, but its effects and specific mechanisms on CME are not fully known. Hence, our study investigated whether puerarin pretreatment could alleviate cardiomyocyte apoptosis and improve cardiac function following CME. The molecular mechanism associated was also explored. A total of 48 Sprague-Dawley rats were randomly divided into $\mathrm{CME}, \mathrm{CME}+$ Puerarin (CME + Pue), sham, and sham + Puerarin (sham + Pue) groups (with 12 rats per group). A CME model was established in CME and CME + Pue groups by injecting $42 \mu \mathrm{m}$ microspheres into the left ventricle of rats. Rats in the CME + Pue and sham + Pue groups were intraperitoneally injected with puerarin at $120 \mathrm{mg} / \mathrm{kg}$ daily for 7 days before operation. Cardiac function, myocardial histopathology, and cardiomyocyte apoptosis index were determined via cardiac ultrasound, hematoxylin-eosin (H\&E) and hematoxylin-basic fuchsin-picric acid (HBFP) stainings, and TdT-mediated dUTP nick-end labeling (TUNEL) staining, respectively. Western blotting was used to measure protein expression related to the phosphoinositide 3-kinase (PI3K)/protein kinase B (Akt)/glycogen synthase kinase-3 $\beta$ (GSK-3 $\beta$ ) pathway. We found that, puerarin significantly ameliorated cardiac dysfunction after $\mathrm{CME}$, attenuated myocardial infarct size, and reduced myocardial apoptotic index. Besides, puerarin inhibited cardiomyocyte apoptosis, as revealed by decreased Bax and cleaved caspase-3, and up-regulated Bcl-2 and PI3K/Akt/GSK-3 $\beta$ pathway related proteins. Collectively, puerarin can inhibit cardiomyocyte apoptosis and thus attenuate myocardial injury caused by CME. Mechanistically, these effects may be achieved through activation of the PI3K/Akt/GSK-3 $\beta$ pathway.
\end{abstract}

\section{INTRODUCTION}

Coronary microembolization (CME) occurs during thrombolytic therapy or percutaneous coronary intervention (PCI) due to formation of plaque debris, small thrombus, or spontaneous rupture of unstable atherosclerotic plaques which block the distal coronary artery microvessels [1,2]. Myocardial slow- or no- reflow phenomena caused by CME triggers myocardial systolic dysfunction, thus may be a powerful independent predictive factor for both long-term adverse prognosis and major cardiovascular adverse events [3]. Studies have shown that microinfarction areas appeared in local myocardium after CME modelling, and apoptotic and necrotic cells developed in cardiomyocytes [4]. Myocardial apoptosis is a crucial factor in CME-induced myo-

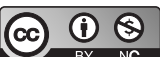

This is an Open Access article distributed under the terms of the Creative Commons Attribution Non-Commercial License, which permits unrestricted non-commercial use, distribution, and reproduction in any medium, provided the original work is properly cited. Copyright @ Korean J Physiol Pharmacol, pISSN 1226-4512, elSSN 2093-3827
Author contributions: Z.C. and Y.Z. conceived and designed research. Z.C., F.C., and J.H. conducted experiments. J.Z. contributed new reagents. H.L. and T.L. contributed analytical tools. Y.Z. and L.L. analyzed data. Z.C. wrote the manuscript. All authors read and approved the manuscript. 
cardial injury and cardiac systolic dysfunction. Therefore, inhibition of myocardial apoptosis may be an effective approach to alleviate myocardial injury following CME [5,6]. Besides, studies have reported that inhibition of phosphatase and tensin homolog may suppress myocardial apoptosis hence ameliorate myocardial systolic function through activation of phosphoinositide 3-kinase (PI3K)/protein kinase B (Akt) pathway [7]. Moreover, pretreatment with drugs such as nicorandil or ligustrazine alleviated CME-induced myocardial injury and myocardial apoptosis, through the PI3K/Akt pathway [8,9].

Puerarin (chemical structure is shown in Fig. 1) is an isoflavonoid extracted from Kudzu root, a Chinese herb. It exhibits various pharmacological activities and hence used to treat cardiovascular diseases in China with minimal associated side effects [10]. Recent studies have shown that puerarin has multiple effects on various cardiovascular diseases, such as anti-apoptosis [11], anti-inflammation [12], anti-oxidative stress [13], anti-platelet aggregation [14]. It also improves myocardial microcirculation [15]. However, whether it can alleviated myocardial apoptosis effects due to CME and ameliorated cardiac functions have not been extensively noted. Besides, studies have reported apoptosis inhibition by puerarin through PI3K/Akt pathway activation $[16,17]$. Hence, in-depth knowledge of the relationship between puerarin and myocardial apoptosis induced by CME in rats is required to manage the CME treatment and prevention. Therefore, the study aims at investigating the roles of puerarin application in myocardial apoptosis and PI3K/Akt/glycogen synthase kinase-3 $\beta$ (GSK$3 \beta$ ) pathway to ascertain molecular mechanism potential on myocardial injury after CME.

\section{METHODS}

\section{Animal preparation}

Experimentally, 48 healthy Sprague-Dawley rats (male, 8 weeks, 250-300 g) were supplied from the medical animal experiment center of Guangxi Medical University. The rats were placed

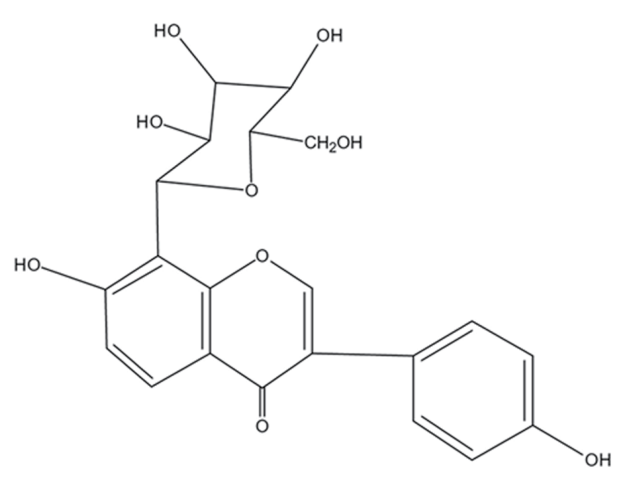

Fig. 1. The chemical structure of puerarin. in a 12-h dark/12-h light circulation chamber with qualified tap water and rat food with maintained humidity $(50 \%-60 \%)$ and temperature $\left(23^{\circ} \mathrm{C} \pm 2^{\circ} \mathrm{C}\right)$ before the experiment. The procedures for animal experiments were approved by the Ethics Committee for Animal Use of Guangxi Medical University and based on the Guidelines for Care and Use of Laboratory Animals.

\section{Coronary microembolization (CME) model establishment and experimental grouping}

From our previous research protocol [18], the CME rat-model was successfully established. The rats were anesthetized by intraperitoneal injection of pentobarbital sodium $(30-40 \mathrm{mg} / \mathrm{kg})$. Subsequently, a tracheotomy was performed to insert a small animal ventilator for respiration, and a left thoracotomy was conducted in the third and fourth intercostal spaces. The ascending aorta was separated and then clamped with a vascular clamp (10 sec), while $0.1 \mathrm{ml}$ of physiological saline suspension with three thousand $42 \mu \mathrm{m}$ polyethylene microspheres (BioSphere Medical Inc., Rockland, MA, USA) was injected into the left ventricle by a microinjector. The rat's chest was closed-up layer-wise and the endotracheal tube was removed after stabilization of their heartbeat and respiration. Thereafter, each rat was intraperitoneally injected with 800,000 IU penicillin. The sham and sham + Pue groups subjected to a similar procedure, but the only difference from the CME operation was that the two groups were injected with an equal amount of $0.1 \mathrm{ml}$ physiological saline instead of microspheres. Herein, the 48 rats were randomly selected into sham, sham + Puerarin (sham + Pue), CME, and CME + Puerarin (CME + Pue) groups (12 rats per group). Rats in the sham + Pue and $\mathrm{CME}+$ Pue groups were intraperitoneally injected with puerarin $(120 \mathrm{mg} / \mathrm{kg} / \mathrm{d})$ for 7 days before operation. The 4 groups recorded no deaths, $12 \mathrm{~h}$ post-surgery.

\section{Cardiac function detection}

Based on our aforementioned research, the cardiac function of rats was worst at $12 \mathrm{~h}$ following CME induction [19], therefore, this time spot was chosen to measure the heart function. The Hewlett Packard Sonos 7500 ultrasonic testing device with a 12 MHz probe (Philips Technologies, Andover, MA, USA) was used to measure the following cardiac function parameters in rats: left ventricular end-systolic diameter (LVESd), fractional shortening (FS), left ventricular end-diastolic diameter (LVEDd) as well as left ventricular ejection fraction (LVEF). The measurements were based on 3 cardiac cycles mean. Besides, a professional physician examined the rats echocardiogram.

\section{Tissue sampling and sample processing}

Herein, $10 \%$ potassium chloride $(2-3 \mathrm{ml})$ was injected through the tail vein to arrest the heart in diastole after the cardiac func- 
tion was determined. Thereafter, the heart was immediately isolated, auricular appendages and atrial parts were removed. Subsequently, cold physiological saline was infused into the ventricles until the rinse solution was colorless. In this step, the ventricle was segmented into apex and base, in the middle of the left ventricle long axis and parallel with the atrioventricular groove. The apex was immediately frozen in the liquid nitrogen earlier prepared, and stored in a refrigerator $\left(-80^{\circ} \mathrm{C}\right)$ for subsequent western blotting. For the heart base, they were fixed with $4 \%$ paraformaldehyde, which lasts about $12 \mathrm{~h}$ for the next paraffin embedding. The tissues were sliced to equal thickness of $4 \mu \mathrm{m}$ for subsequent pathological examination, including hematoxylin-eosin (H\&E) staining, hematoxylin-basic fuchsin-picric acid (HBFP) staining, and TdT-mediated dUTP nick-end labeling (TUNEL) staining.

\section{Measurement of myocardial microinfarct areas}

The HBFP staining is an important staining method in diagnosing early myocardial ischemia. With this technique, the nucleus and cytoplasm of normal cardiomyocytes are stained blue and yellow, respectively, whereas ischemic cardiomyocytes and red blood cells are stained red. The HBFP-stained slices were observed ( $\times 100$ magnifications) under a pathological image analyzer (DMR + Q550; Leica, Wetzlar, Germany). Accordingly, 5 microscopic visual fields were randomly selected from HBFPstained slices to observe and analyze the area of myocardial infarction using the Leica Qwin software and planar area method. The infarct percentage was determined by dividing the ischemic area by the gross observed area [20].

\section{Detection of cardiomyocyte apoptosis by TUNEL staining}

Following the manufacturer's protocol, TUNEL staining was conducted to determine cardiomyocyte apoptosis by using commercial kits (Roche, Indianapolis, IN, USA). The nuclei of normal cardiomyocytes without apoptosis were stained light blue, whereas apoptotic cardiomyocytes were stained yellow-brown (TUNEL positive) under the light microscope. Precisely 20 non-overlapping areas ( $\times 400$ magnifications) in each section were randomly selected to determine the number of apoptotic cardiomyocytes, total cardiomyocytes in the micro-infarct area, marginal infarct area, and distal infarct area. The apoptosis index (AI) was based on the formula: $\mathrm{AI}=$ (number of apoptotic cardiomyocytes / total number of cardiomyocytes) $\times 100 \%$ [21].

\section{Western blot analysis}

The rat myocardial tissue total proteins were extracted with protein lysis buffer, and its concentration was detected with the bicinchoninic acid (BCA) assay kit. An equal amount of protein was separated by $10 \%-15 \%$ sodium dodecyl sulphate-polyacryl- amide gel electrophoresis before transferred onto PVDF (polyvinylidene fluoride) membranes (Millipore, Atlanta, GA, USA). Consequently, the PVDF membranes were blocked at room temperature for about 1 hour with $5 \%$ bovine serum albumin or nonfat milk. The membranes were incubated overnight at a stable temperature of $4^{\circ} \mathrm{C}$ with primary antibodies as follows: phospho-

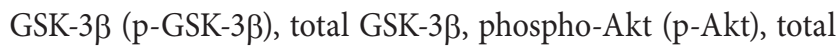
Akt, phospho-PI3K (p-PI3K), total PI3K, cleaved caspase-3, Bcl-2, Bax, or GAPDH (diluted concentration of primary antibodies was 1:1,000). The primary antibodies against the total PI3K, p-PI3K, $\mathrm{Bax}$, and Bcl-2 were purchased from Abcam (Cambridge, UK) whereas the primary antibodies against the cleaved caspase-3, p-Akt, total Akt, total GSK-3 $\beta$, p-GSK-3 $\beta$, and GAPDH were acquired from Cell Signaling Technology (Beverly, MA, USA). Thereafter, the membranes were rinsed 5 times with TBST and incubated at room temperature for $2 \mathrm{~h}$ with horseradish peroxidase-conjugated secondary antibodies prepared in advance. An enhanced chemiluminescence detection device (Pierce, Rockford, IL, USA) was used to assess the protein signals. Protein bands were evaluated and quantified with the ImageJ software (National Institutes of Health, Bethesda, MD, USA).

\section{Statistical analysis}

The SPSS 23.0 (IBM Co., Armonk, NY, USA) software for statistical analysis was used for the quantitative data expressed as mean \pm standard deviation. Comparisons were performed using the Student's t-test between two groups or using the one-way analysis of variance (ANOVA) followed by Student-NewmanKeuls post-hoc tests for multiple groups. $\mathrm{p}<0.05$ indicates statistical significance.

\section{RESULTS}

\section{Puerarin ameliorated cardiac function following CME}

The echocardiographic examination results in the 4 groups are summarized in Fig. 2. To be brief, compared to the sham group, the CME group could cause significant cardiac dysfunction, which was mainly reflected by reduced LVEF and LVFS, but increased LVEDd and LVESd ( $\mathrm{p}<0.05)$. However, compared to the CME group, the CME + Pue group demonstrated an apparent improvement in cardiac function following CME induction, as manifested by an evident increase in LVEF and LVFS, but a significant decrease in LVEDd and LVESd $(p<0.05)$. Both sham and sham + Pue groups showed insignificant $(p>0.05)$ cardiac functions. Accordingly, these results showed puerarin pretreatment could distinctly ameliorate cardiac function following CME. 
A

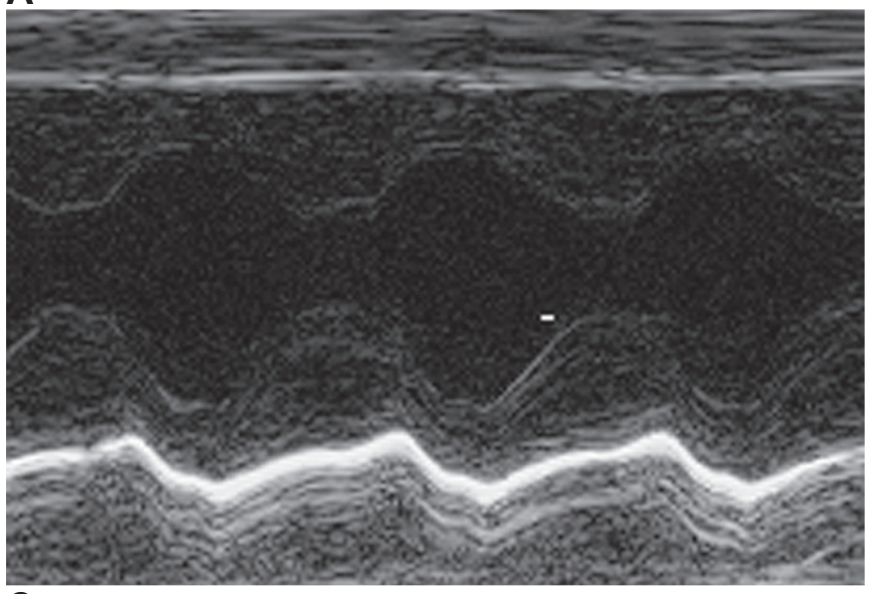

c
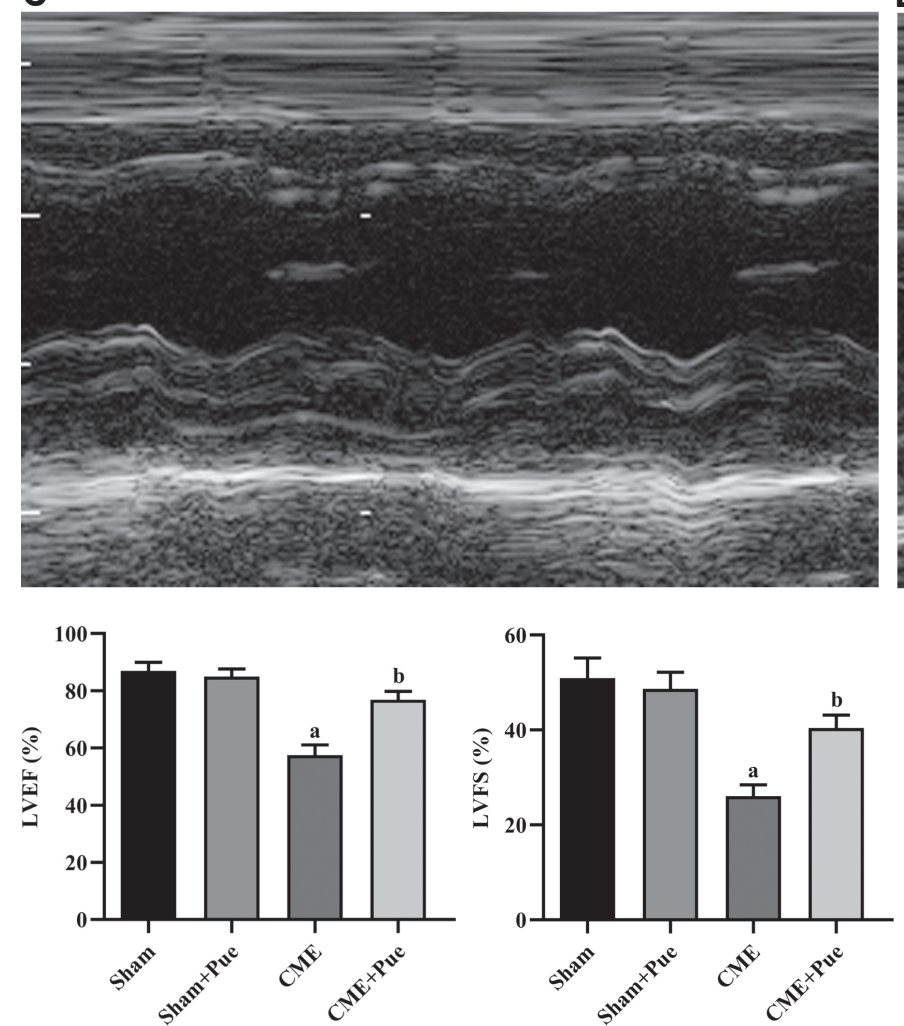

B

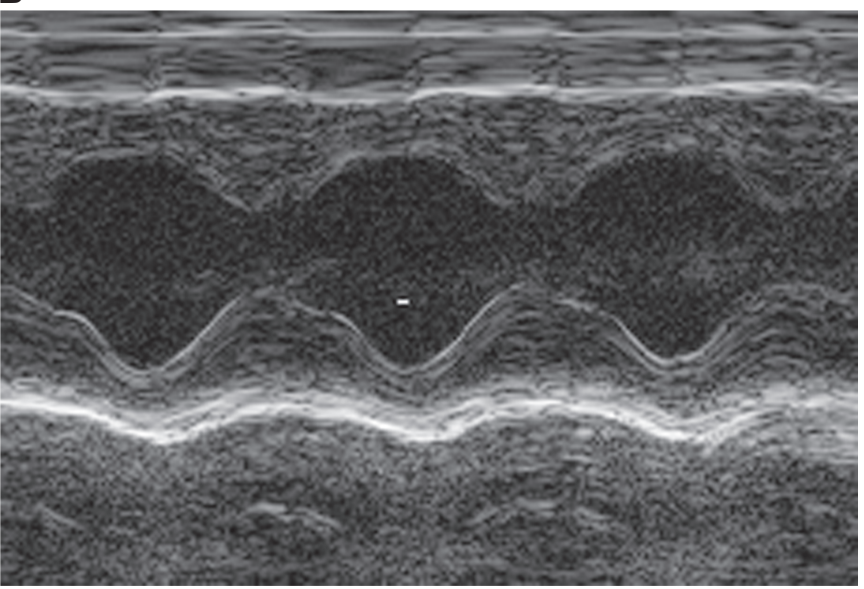

D

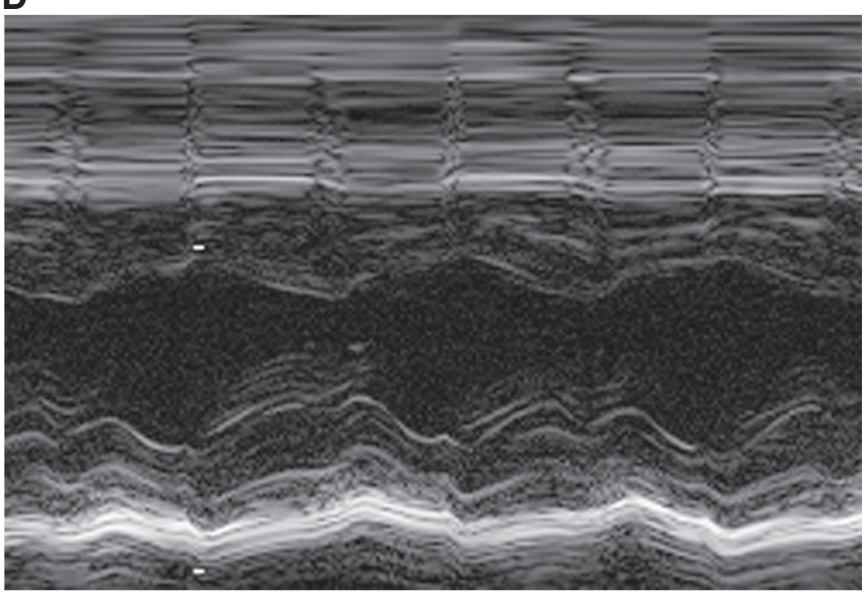

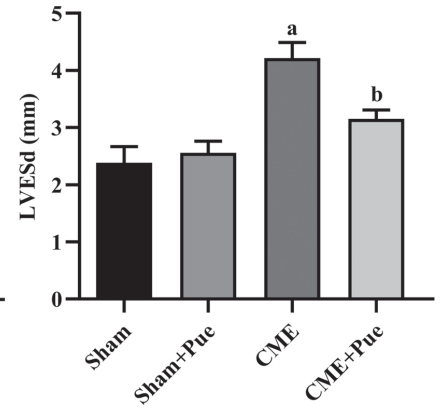

Fig. 2. Echocardiographic analysis of rats in four groups. (A-D) Sham, sham + Pue, CME, CME + Pue group, respectively. Left ventricular fractional shortening (LVFS), left ventricular end-diastolic diameter (LVEDd), left ventricular ejection fraction (LVEF), and left ventricular end-systolic diameter (LVESd) were quantitatively analyzed. CME, coronary microembolization; Pue, puerarin. ${ }^{a} p<0.05$ in comparison with the sham group; ${ }^{b} p<0.05$ in comparison with the CME group.

\section{CME histopathology}

H\&E staining and HBFP staining showed that subendocardial ischemia was occasionally found in the sham group and sham + Pue group, but no obvious infarction was seen. However, multiple microinfarctions were observed both in the CME and CME + Pue groups. The HBFP staining exhibited that most microinfarct regions were wedge-shaped with a localized distribution and common in the subendocardial and left ventricle (Fig. 3).
From the H\&E staining results, the nucleus of cardiomyocytes in CME group dissolved or disappeared in the micro-infarct zones, with cytoplasmic red staining. Moreover, peripheral myocardial edema, peripheral inflammatory cell infiltration, red blood cell exudation, and microembolism in arteriole were observed in the microinfarct areas. However, pretreatment with puerarin could significantly improve these conditions, including the reduction of peripheral myocardial edema and inflammatory cell infiltration (Fig. 4). The infarct areas of the sham group, sham + Pue group, 


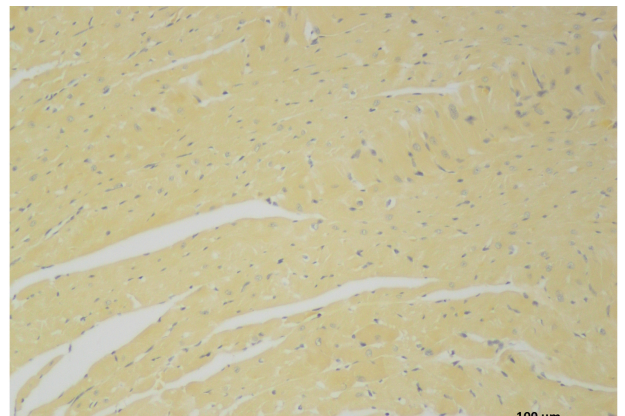

A
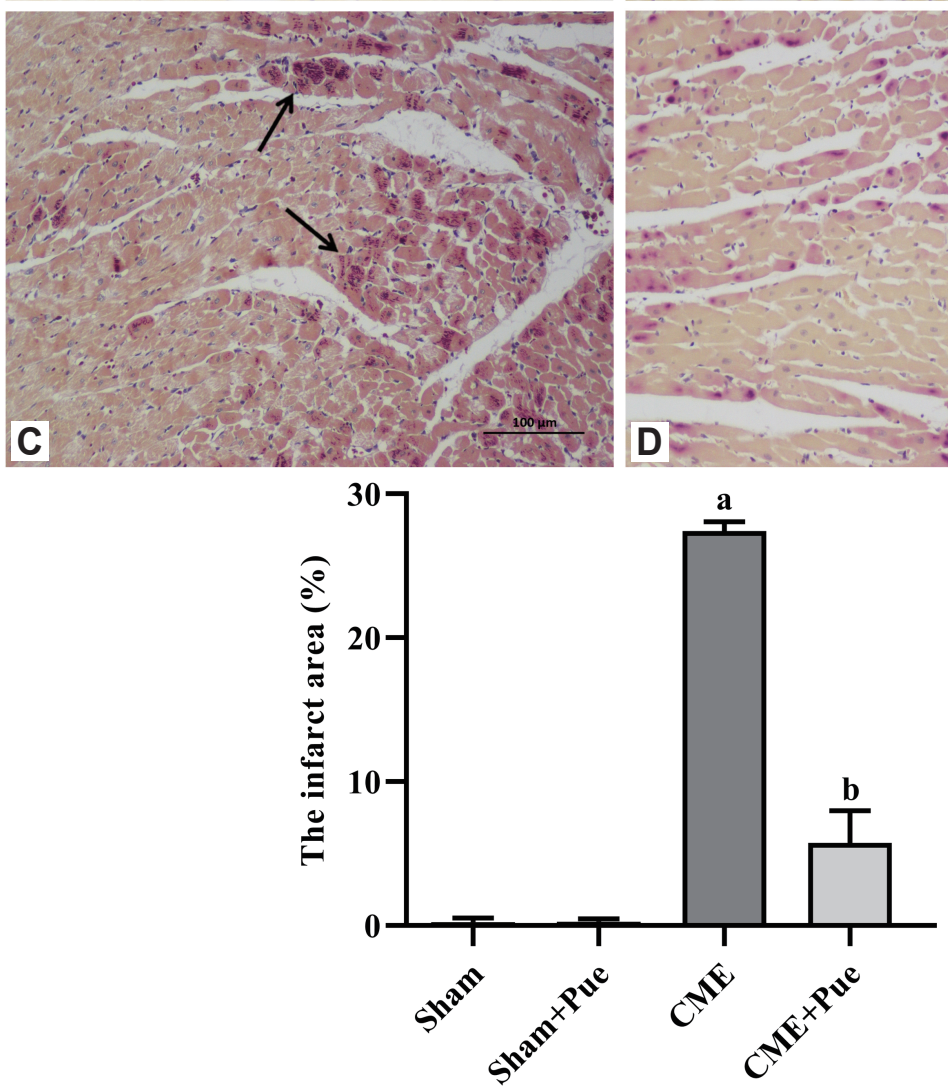
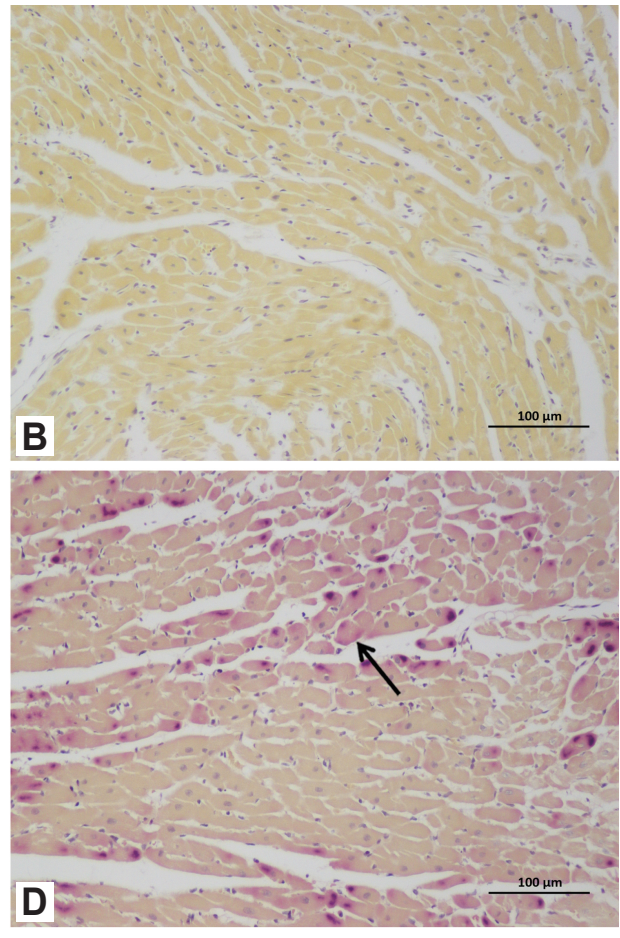

ra . 

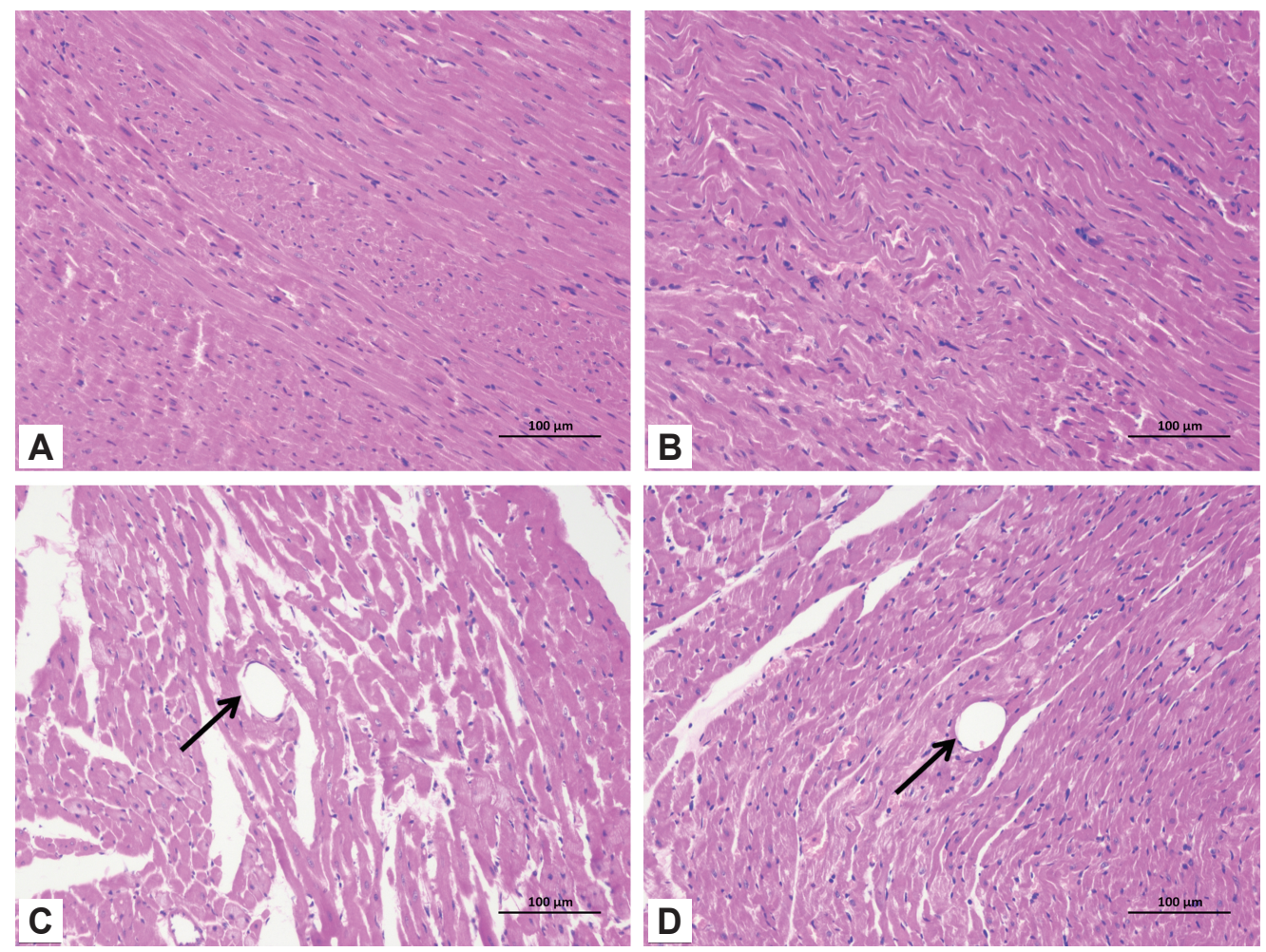

Fig. 4. Histopathological examination of myocardial tissue by H\&E staining. (A-D) Sham, sham + Pue, CME, CME + Pue group, respectively. Microspheres with inflammatory cells infiltration were detected in both the CME and CME + Pue groups, but these pathological findings were not observed in the sham and sham + Pue groups. The arrows in (C and D) indicate microspheres. CME, coronary microembolization; Pue, puerarin.

\section{Puerarin reduced cardiomyocyte apoptosis by regulating cleaved caspase-3, $\mathrm{Bax}$ and $\mathrm{BCl}-2$ protein levels after CME}

The cleaved caspase-3, Bax, and Bcl-2 protein levels in each group were assessed to confirm myocardial apoptosis following CME. Notably, the Bcl-2/Bax ratio, in comparison with the sham group, was significantly reduced (Fig. 6), but the expression of cleaved caspase-3 was increased in the CME group ( $\mathrm{p}<$ 0.05). However, in the CME + Pue group, puerarin pretreatment distinctly up-regulated the $\mathrm{Bcl}-2 / \mathrm{Bax}$ ratio and down-regulated cleaved caspase- 3 protein expression as compared to the CME group $(\mathrm{p}<0.05)$. There was no significant difference in the expression of cleaved caspase- 3 and the ratio of $\mathrm{Bcl}-2 / \mathrm{Bax}$ between the sham group and the sham + Pue group ( $p>0.05)$. Thus, the results suggested that puerarin administration can alleviate CME-induced cardiomyocyte apoptosis.

\section{Effects of puerarin on protein levels of the PI3K/Akt/ GSK-3 $\beta$ pathway}

Western blot was conducted in each group to detect the relative levels of the PI3K/Akt/GSK-3 $\beta$ pathway (Fig. 7). The relative protein levels of total GSK-3 $\beta$, total Akt, and total PI3K in the four groups were not significantly different $(p<0.05)$. In comparison with the sham group, myocardial levels of p-GSK-3 $\beta$, p-Akt as well as $\mathrm{p}$-PI3K in the CME group showed a distinct decrease ( $\mathrm{p}$ $<0.05$ ). However, compared with the CME group, after pretreatment with puerarin, myocardial p-GSK-3 $\beta$, p-Akt, and p-PI3K levels in the CME + Pue group showed a distinct increase $(\mathrm{p}<$ 0.05). The expression of p-GSK-3 $\beta$, p-Akt, and p-PI3K between the sham group and the sham + Pue group were not significantly different $(\mathrm{p}>0.05)$.

\section{DISCUSSION}

From our research findings, we showed that myocardial apoptosis increased significantly in rats following CME modeling. Consequently, it led to both cardiac dysfunction and myocardial injury while the PI3K/Akt/GSK-3 $\beta$ pathway was closely related to CME-induced cardiomyocyte apoptosis. Notably, puerarin pretreatment 7 days before CME induction could significantly inhibit myocardial apoptosis and thus attenuate myocardial injury and improve cardiac function. Besides, the study indicated the role of puerarin in protecting the heart from myocardial injury induced by CME may be achieved through the PI3K/Akt/GSK$3 \beta$ signaling pathway activation. These findings mentioned above highlighted the potential role and significance of PI3K/Akt/GSK$3 \beta$ signaling pathway in the pathogenesis of cardiomyocyte apoptosis induced by CME. For the first time, our study demonstrated that the administration of puerarin before CME may ameliorate 

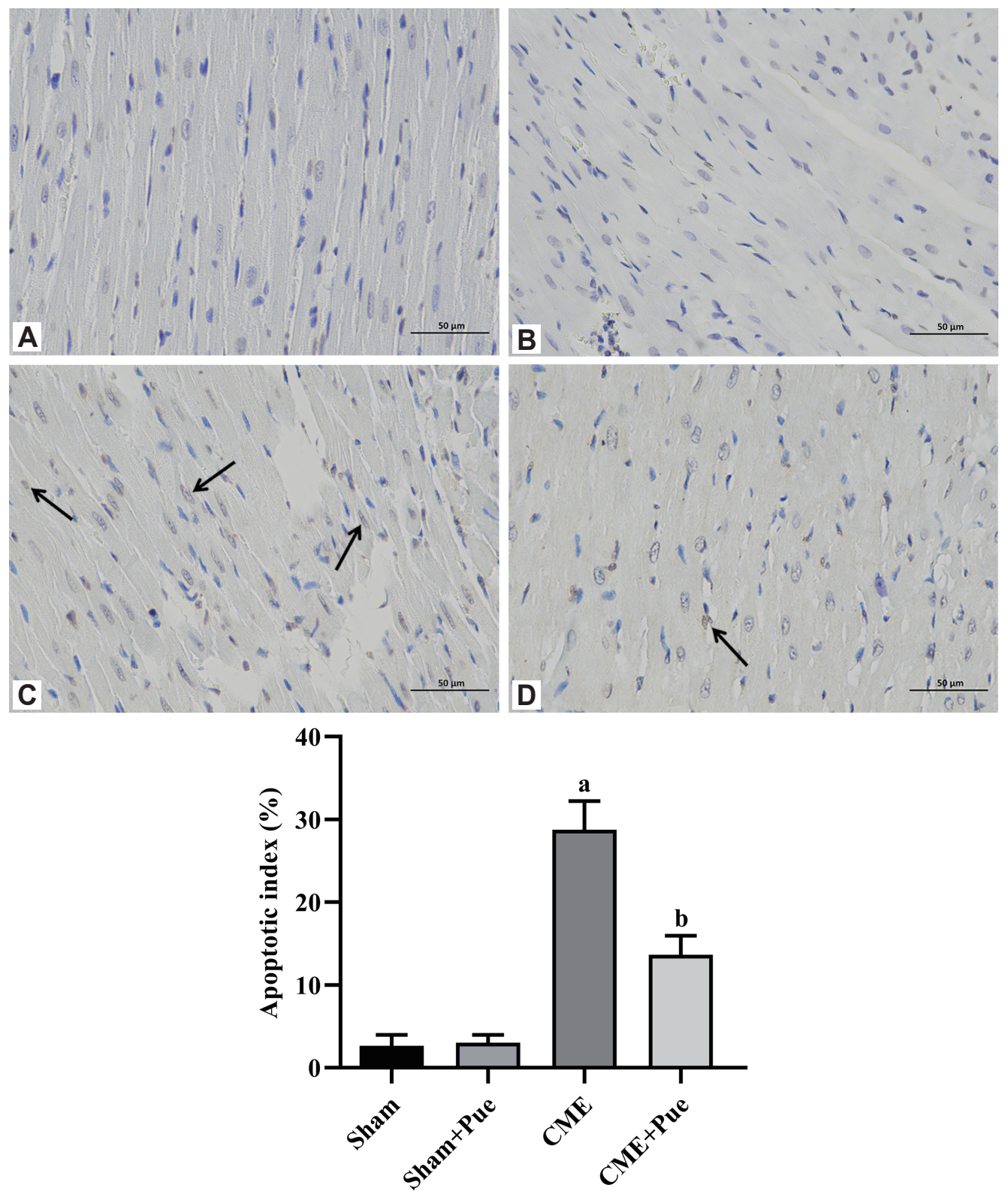

Fig. 5. TUNEL staining of myocardial apoptosis in the four groups. (A-D) Sham, sham + Pue, CME, CME + Pue group, respectively. Data are expressed as mean \pm standard deviation. Nuclei of apoptotic cardiomyocytes were yellow-brown, whereas the normal cardiomyocytes that did not show apoptosis were light blue. The arrows in ( $C$ and $D)$ indicate the nuclei of apoptotic cardiomyocytes. CME, coronary microembolization; Pue, puerarin. ${ }^{a} p<0.05$ in comparison with the sham group; ${ }^{b} p<0.05$ in comparison with the CME group.

cardiac dysfunction and alleviate myocardial injury through regulating the PI3K/Akt/GSK-3 $\beta$ pathway. Accordingly, we proposed puerarin pretreatment as a potential treatment strategy for myocardial injury caused by CME.

As a serious complication, the CME occurs during a selective coronary intervention or primary percutaneous coronary intervention [22]. Notably, left ventricular dysfunction caused by CME differs from proximal vascular occlusion in epicardium and is not tightly relevant to the degree of the myocardial perfusion defect. Simply, micro-infarct areas or lack of local myocardial perfusion cannot explain this phenomenon. Studies by Dörge et al. [23] showed that coronary blood flow in dogs was shortly decreased after microspheres were injected into the coronary arteries, whereas cardiac contractile function was gradually damaged. Besides, both pigs and dogs CME were studied and revealed that the proportion of the microinfarct area was less than $5 \%$ of the gross myocardium [24], which cannot satisfactorily interpret the progressive cardiac systolic dysfunction. Instead, myocardial apoptosis around the microinfarct areas was considered to be the pivotal factor of progressive myocardial contractile dysfunction and myocardial injury following CME $[25,26]$. Thus, our CME rat models demonstrated aggravated cardiac dysfunction and cardiomyocyte apoptosis in the peri-infarct and microinfarct regions, which concur with pathophysiological changes in CME. 

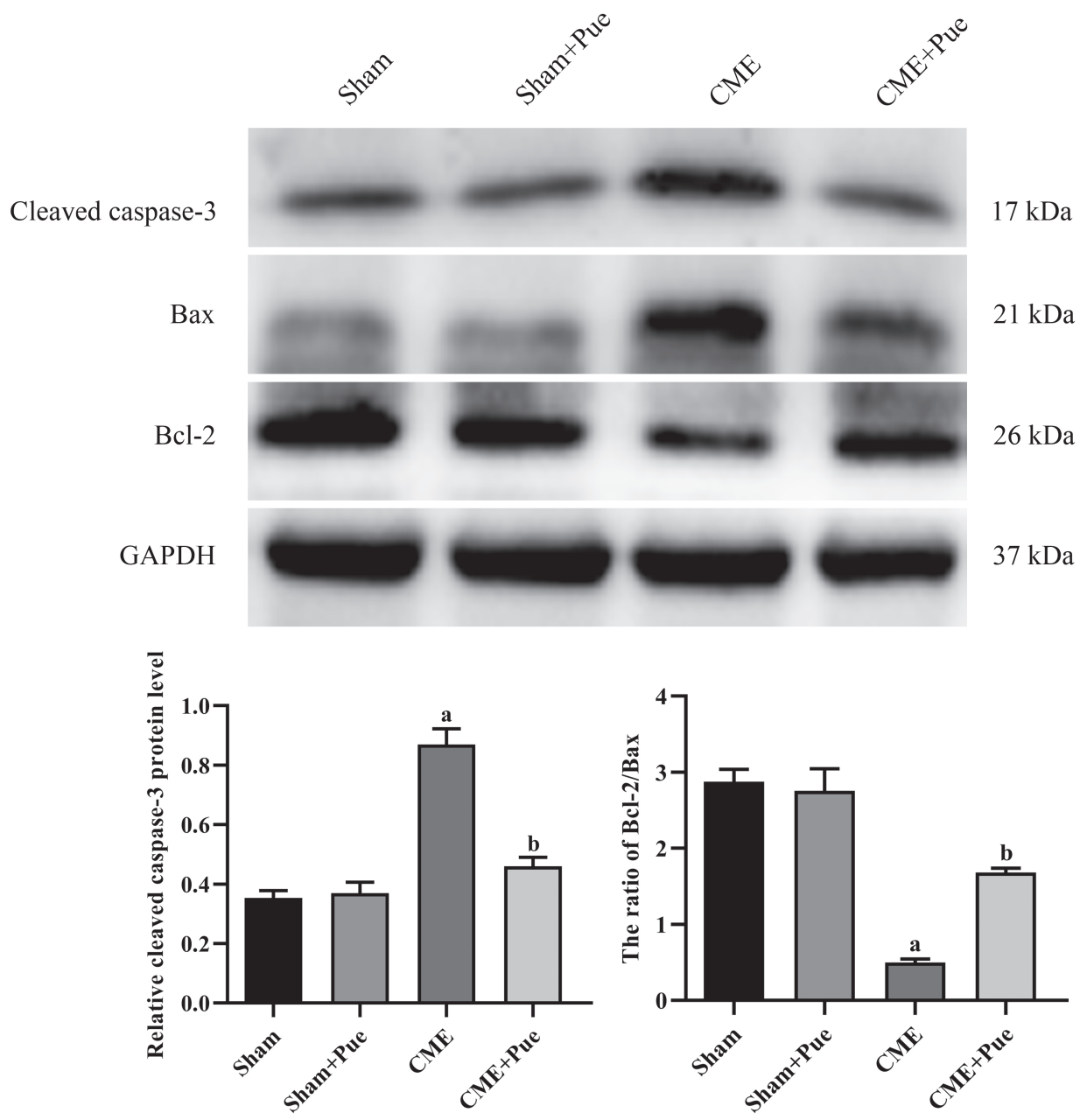

Fig. 6. Puerarin reduced cardiomyocyte apoptosis by regulating cleaved caspase-3, Bax as well as Bcl-2 after CME. Values are presented as mean \pm standard deviation. The data were determined by at least three independent experiments in the present study. CME, coronary microembolization; Pue, puerarin. ${ }^{a} p<0.05$ in comparison with the sham group; ${ }^{b} p<0.05$ in comparison with the CME group.

Puerarin is the main and very effective monomer ingredient of Pueraria radix, which is a traditional Chinese medicine [27]. Puerarin is well known for its multiple pharmacological effects, including improving microcirculation, dilating blood vessels as well as its anti-oxidative stress, anti-apoptotic and anti-inflammatory properties, and is therefore widely used to manage cardiovascular and cerebrovascular diseases in China [28,29]. In a severe burninduced rat acute myocardial injury model, studies had shown puerarin treatment can improve the ultrastructure changes in cardiomyocytes, decrease malondialdehyde (MDA) content, inhibit cardiac myeloperoxidase activity, reduce tumor necrosis factor- $\alpha$ concentration in serum, block the activation of $\mathrm{p} 38$ mitogen-activated protein kinase to attenuate oxidative stress and alleviate inflammatory responses in the heart [30]. According to Guo et al. [31] study, puerarin treatment significantly decreased MDA activity, increased superoxide dismutase activity, and reduced caspase-3 activity in the diabetic myocardial ischemia/reperfusion rat model, thereby effectively reducing oxidative stress and attenuating myocardial apoptosis. Puerarin administration effectively inhibiting the apoptosis of neurocytes through the PI3K/Akt pathway activation following cerebral ischemia/reperfusion injury in rats was reported by Han et al. [32]. Moreover, Li et al. [16] demonstrated that puerarin pretreatment can attenuate daunorubicin-induced $\mathrm{H} 9 \mathrm{c} 2$ cell apoptosis, which is reflected by the inhibition of extracellular $\mathrm{Ca}^{2+}$ influx and the decrease of cleaved caspase- 3 protein level, although it increased the p-Akt protein level. However, these effects were reversed by LY294002, a specific inhibitor of $\mathrm{P} 13 \mathrm{~K}$, suggesting that puerarin inhibits apoptosis and exerts cardioprotective effects via activation of the PI3K/ Akt signaling pathway. Based on these results, we proposed that puerarin pretreatment may have the potential to protect the heart from CME-induced myocardial injury by regulating PI3K/Akt signaling pathway to reduce myocardial apoptosis.

Accordingly, the PI3K/Akt signaling pathway was found to exert an indispensable effect on cardiomyocyte apoptosis and myocardial injury caused by CME [33,34]. The PI3K/Akt signaling pathway involves multiple intracellular signal transduction mediators and effector proteins while GSK-3 $\beta$ is one of its downstream regulatory mediators [35]. Activated Akt promotes phosphorylation of serine 9 at GSK-3 $\beta$, thereby significantly inhibiting its biological activity, ultimately suppressing the opening of mitochondrial permeability transition pore and promoting cell survival [36-38]. The PI3K/Akt/GSK-3 $\beta$ pathway, as an essential signal transduction pathway, plays a very significant and integral 


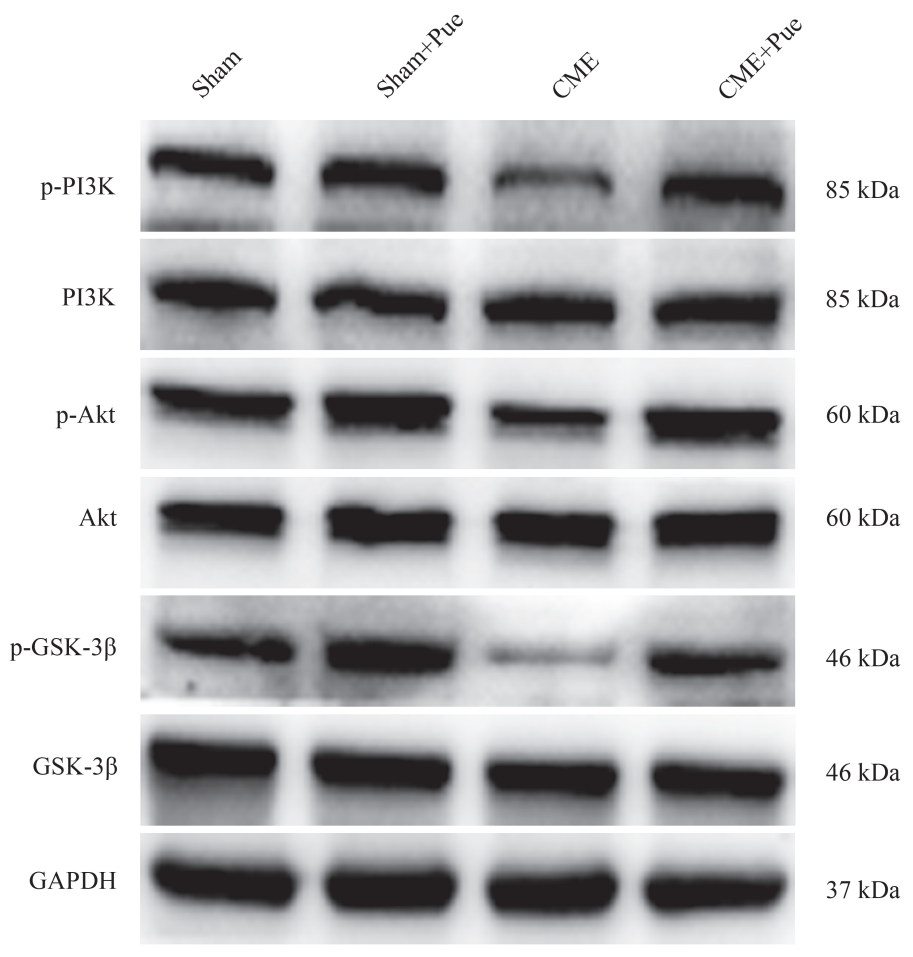

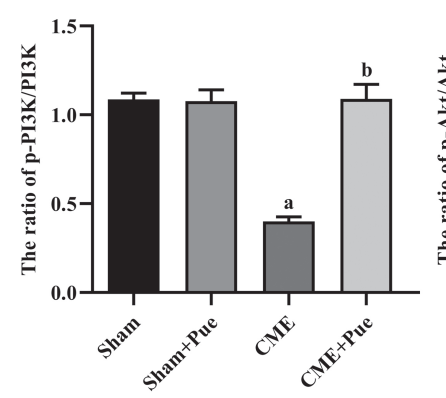

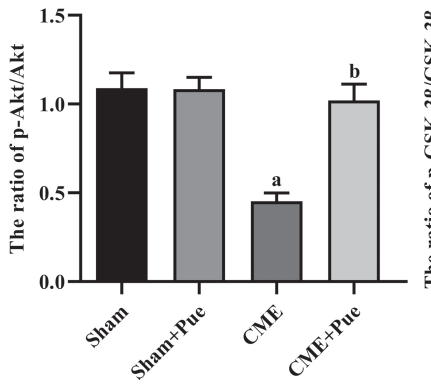

biological role in apoptosis, cell proliferation, and survival [35,39]. Studies have indicated that activation of the PI3K/Akt/GSK-3 $\beta$ signaling pathway can significantly alleviate myocardial injury and cardiomyocyte apoptosis, but its inactivation results in opposite effects [40,41]. In the present research, notably, we showed that puerarin pretreatment ameliorated cardiac function, attenuated myocardial infarction area, decreased cardiomyocyte apoptosis index, and thus reduced myocardial injury caused by CME. Also, puerarin could significantly down-regulate Bax and cleaved caspase-3 proteins, but distinctly up-regulate the levels of $\mathrm{Bcl}-2$, $\mathrm{p}$ P13K, p-Akt, and p-GSK-3 $\beta$. Cumulatively, the results indicated the potential benefits of puerarin pretreatment for CME-induced myocardial injury by activating PI3K/Akt/GSK-3 $\beta$ pathway, thus inhibiting cardiomyocyte apoptosis and improving cardiac dysfunction.

This work has the following limitations. First, in our previous study, the heart function of rats was the worst at $12 \mathrm{~h}$ after CME modeling, so this research solely focused on this time point to observe the effect of puerarin on CME. Therefore, whether puerarin can continue to significantly improve cardiac function,

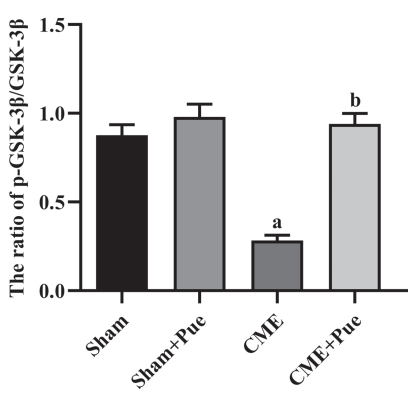

Fig. 7. Effects of puerarin on PI3K/Akt/ GSK-3 $\beta$ pathway. Values are expressed as mean \pm standard deviation. The data were determined by at least three independent experiments in the present study. CME, coronary microembolization; Pue, puerarin. ${ }^{a} \mathrm{p}<0.05$ in comparison with the sham group; ${ }^{b} p<0.05$ in comparison with the CME group.

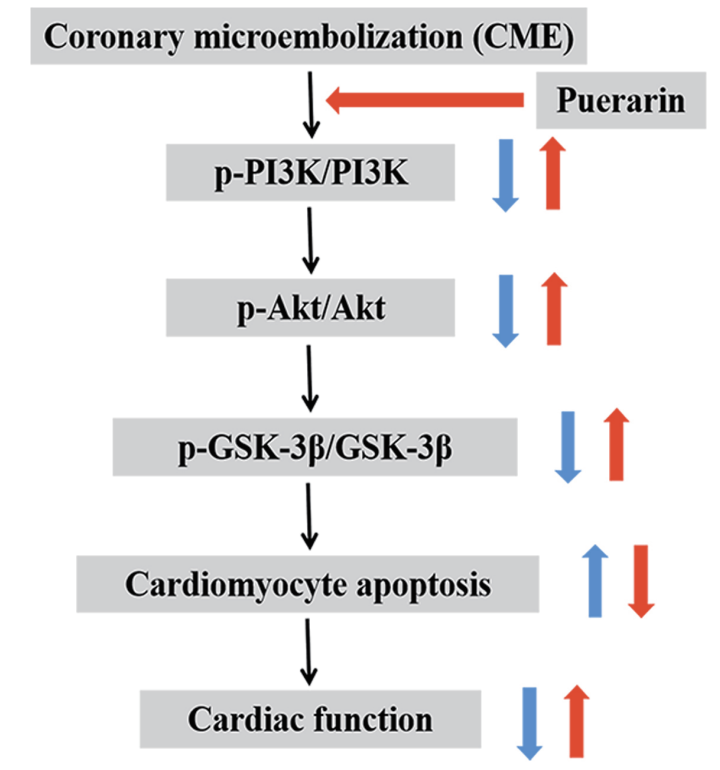

Fig. 8. The graphical abstract shows that puerarin inhibits cardiomyocyte apoptosis induced by coronary microembolization in rats by activating the PI3K/Akt/GSK-3 $\beta$ signaling pathway. 
inhibit cardiomyocyte apoptosis, and reduce myocardial injury more than $12 \mathrm{~h}$ after CME operation remains elusive. Secondly, this study demonstrated that puerarin pretreatment may inhibit cardiomyocyte apoptosis and alleviate myocardial injury via the PI3K/Akt/GSK-3 $\beta$ signaling pathway activation, but no specific intervention against PI3K/Akt/GSK-3 $\beta$ signaling pathway was used after puerarin administration to further confirm the molecular mechanism of puerarin on CME. Therefore, future studies are recommended to block the PI3K/Akt/GSK-3 $\beta$ pathway using PI3K inhibitors, such as LY294002, which can provide more reliable support for elucidating the role of puerarin in CME and its specific molecular mechanism.

Conclusively, puerarin administration can significantly improve cardiac function and attenuate myocardial injury induced by CME, which may be exerted by a notable down-regulation of cardiomyocyte apoptosis via the PI3K/Akt/GSK-3 $\beta$ pathway activation (summarized in Fig. 8, graphical abstract). As a result, our study highlights the potential clinical importance of puerarin pretreatment in CME treatment, which has significant value for the PCI-treatment patients.

\section{ACKNOWLEDGEMENTS}

This study was supported by the National Natural Science Foundation of China (Grant No. 81770346) and the Project for Innovative Research Team in Guangxi Natural Science Foundation (Grant No. 2018GXNSFGA281006).

\section{CONFLICTS OF INTEREST}

The authors declare no conflicts of interest.

\section{REFERENCES}

1. Heusch G, Skyschally A, Kleinbongard P. Coronary microembolization and microvascular dysfunction. Int J Cardiol. 2018;258:17-23.

2. Scarpone M, Cenko E, Manfrini O. Coronary no-reflow phenomenon in clinical practice. Curr Pharm Des. 2018;24:2927-2933.

3. Srinivasan M, Rihal C, Holmes DR, Prasad A. Adjunctive thrombectomy and distal protection in primary percutaneous coronary intervention: impact on microvascular perfusion and outcomes. Circulation. 2009;119:1311-1319.

4. He WK, Su Q, Liang JB, Wang XT, Sun YH, Li L. Nicorandil pretreatment inhibits myocardial apoptosis and improves cardiac function after coronary microembolization in rats. J Geriatr Cardiol. 2018;15:591-597.

5. Xue Y, Sun C, Hao Q, Cheng J. Astaxanthin ameliorates cardiomyocyte apoptosis after coronary microembolization by inhibiting oxidative stress via Nrf2/HO-1 pathway in rats. Naunyn Schmiedebergs Arch Pharmacol. 2019;392:341-348.
6. Liu YC, Li L, Su Q, Liu T, Tang ZL. Trimetazidine pretreatment inhibits myocardial apoptosis and improves cardiac function in a Swine model of coronary microembolization. Cardiology. 2015;130:130-136.

7. Wang J, Chen H, Su Q, Zhou Y, Liu T, Li L. The PTEN/Akt signaling pathway mediates myocardial apoptosis in swine after coronary microembolization. J Cardiovasc Pharmacol Ther. 2016;21:471-477.

8. Su Q, Li L, Zhao J, Sun Y, Yang H. Effects of nicorandil on PI3K/Akt signaling pathway and its anti-apoptotic mechanisms in coronary microembolization in rats. Oncotarget. 2017;8:99347-99358.

9. Su Q, Lv X, Ye Z. Ligustrazine attenuates myocardial injury induced by coronary microembolization in rats by activating the PI3K/Akt pathway. Oxid Med Cell Longev. 2019;2019:6791457.

10. Wei SY, Chen Y, Xu XY. Progress on the pharmacological research of puerarin: a review. Chin J Nat Med. 2014;12:407-414.

11. He L, Wang T, Chen BW, Lu FM, Xu J. Puerarin inhibits apoptosis and inflammation in myocardial cells via PPAR $\alpha$ expression in rats with chronic heart failure. Exp Ther Med. 2019;18:3347-3356.

12. Yin MS, Zhang YC, Xu SH, Liu JJ, Sun XH, Liang C, Wang Y, Li J, Wang FW, Wang QL, Mu YL. Puerarin prevents diabetic cardiomyopathy in vivo and in vitro by inhibition of inflammation. J Asian Nat Prod Res. 2019;21:476-493.

13. Xu HX, Pan W, Qian JF, Liu F, Dong HQ, Liu QJ. MicroRNA-21 contributes to the puerarin-induced cardioprotection via suppression of apoptosis and oxidative stress in a cell model of ischemia/ reperfusion injury. Mol Med Rep. 2019;20:719-727.

14. Liu Q, Lu Z, Wang L. Restrictive effect of puerarin on myocardial infarct area in dogs and its possible mechanism. J Tongii Med Univ. 2000;20:43-45.

15. He H, Shi M, Yang J, Zeng X, Qiao H, Wu L, Li L. The correlation between angiogenesis and abnormal expression of SERCA2a, phospholamban and the endothelin pathway in heart failure, and improvement by puerarin. Phytother Res. 2008;22:948-956.

16. Li W, Lu M, Zhang Y, Xia D, Chen Z, Wang L, Yin N, Wang Z. Puerarin attenuates the daunorubicin-induced apoptosis of $\mathrm{H} 9 \mathrm{c} 2$ cells by activating the PI3K/Akt signaling pathway via the inhibition of $\mathrm{Ca}^{2+}$ influx. Int J Mol Med. 2017;40:1889-1894.

17. Liang F, Xie S. Puerarin prevents tumor necrosis factor- $\alpha$-induced apoptosis of PC12 cells via activation of the PI3K/Akt signaling pathway. Exp Ther Med. 2017;14:813-818.

18. He W, Su Q, Liang J, Sun Y, Wang X, Li L. The protective effect of nicorandil on cardiomyocyte apoptosis after coronary microembolization by activating Nrf2/HO-1 signaling pathway in rats. Biochem Biophys Res Commun. 2018;496:1296-1301.

19. Su Q, Lv X, Sun Y, Ye Z, Kong B, Qin Z. Role of TLR4/MyD88/NF$\kappa \mathrm{B}$ signaling pathway in coronary microembolization-induced myocardial injury prevented and treated with nicorandil. Biomed Pharmacother. 2018;106:776-784.

20. Su Q, Li L, Liu T, Wang J, Zhou Y, Liu Y. Effects of atorvastatin on $\mathrm{PDCD} 4 / \mathrm{NF}-\kappa \mathrm{B} / \mathrm{TNF}-\alpha$ signaling pathway during coronary microembolization of miniature pigs. Exp Mol Pathol. 2015;99:564-569.

21. Mao Q, Liang X, Wu Y, Lu Y. Resveratrol attenuates cardiomyocyte apoptosis in rats induced by coronary microembolization through SIRT1-mediated deacetylation of p53. J Cardiovasc Pharmacol Ther. 2019;24:551-558.

22. Skyschally A, Walter B, Heusch G. Coronary microembolization during early reperfusion: infarct extension, but protection by isch- 
aemic postconditioning. Eur Heart J. 2013;34:3314-3321.

23. Dörge H, Neumann T, Behrends M, Skyschally A, Schulz R, Kasper C, Erbel R, Heusch G. Perfusion-contraction mismatch with coronary microvascular obstruction: role of inflammation. Am J Physiol Heart Circ Physiol. 2000;279:H2587-H2592.

24. Skyschally A, Haude M, Dörge H, Thielmann M, Duschin A, van de Sand A, Konietzka I, Büchert A, Aker S, Massoudy P, Schulz R, Erbel R, Heusch G. Glucocorticoid treatment prevents progressive myocardial dysfunction resulting from experimental coronary microembolization. Circulation. 2004;109:2337-2342.

25. Liang J, Li L, Sun Y, He W, Wang X, Su Q. The protective effect of activating Nrf2/HO-1 signaling pathway on cardiomyocyte apoptosis after coronary microembolization in rats. BMC Cardiovasc Disord. 2017;17:272.

26. Wang XT, Lu YX, Sun YH, He WK, Liang JB, Li L. TAK-242 protects against apoptosis in coronary microembolization-induced myocardial injury in rats by suppressing TLR4/NF- $\kappa \mathrm{B}$ signaling pathway. Cell Physiol Biochem. 2017;41:1675-1683.

27. Zhao GJ, Hou N, Cai SA, Liu XW, Li AQ, Cheng CF, Huang Y, Li LR, Mai YP, Liu SM, Ou CW, Xiong ZY, Chen XH, Chen MS, Luo CF. Contributions of Nrf2 to puerarin prevention of cardiac hypertrophy and its metabolic enzymes expression in rats. J Pharmacol Exp Ther. 2018;366:458-469.

28. Shi W, Yuan R, Chen X, Xin Q, Wang Y, Shang X, Cong W, Chen K. Puerarin reduces blood pressure in spontaneously hypertensive rats by targeting eNOS. Am J Chin Med. 2019;47:19-38.

29. Zhou YX, Zhang H, Peng C. Puerarin: a review of pharmacological effects. Phytother Res. 2014;28:961-975.

30. Liu S, Ren HB, Chen XL, Wang F, Wang RS, Zhou B, Wang C, Sun YX, Wang YJ. Puerarin attenuates severe burn-induced acute myocardial injury in rats. Burns. 2015;41:1748-1757.

31. Guo BQ, Xu JB, Xiao M, Ding M, Duan LJ. Puerarin reduces ischemia/reperfusion-induced myocardial injury in diabetic rats via upregulation of vascular endothelial growth factor A/angiotensin-1 and suppression of apoptosis. Mol Med Rep. 2018;17:7421-7427.

32. Han JQ, Yu KY, He M. [Effects of puerarin on the neurocyte apop- tosis and p-Akt (Ser473) expressions in rats with cerebral ischemia/reperfusion injury]. Zhongguo Zhong Xi Yi Jie He Za Zhi. 2012;32:1069-1072. Chinese.

33. Wang J, Chen H, Zhou Y, Su Q, Liu T, Wang XT, Li L. Atorvastatin inhibits myocardial apoptosis in a swine model of coronary microembolization by regulating PTEN/PI3K/Akt signaling pathway. Cell Physiol Biochem. 2016;38:207-219.

34. Zhu HH, Wang XT, Sun YH, He WK, Liang JB, Mo BH, Li L. MicroRNA-486-5p targeting PTEN protects against coronary microembolization-induced cardiomyocyte apoptosis in rats by activating the PI3K/AKT pathway. Eur J Pharmacol. 2019;855:244-251.

35. Yao Y, Wang Y, Kong L, Chen Y, Yang J. Osthole decreases tau protein phosphorylation via PI3K/AKT/GSK-3 $\beta$ signaling pathway in Alzheimer's disease. Life Sci. 2019;217:16-24.

36. Feng MG, Liu CF, Chen L, Feng WB, Liu M, Hai H, Lu JM. MiR21 attenuates apoptosis-triggered by amyloid- $\beta$ via modulating PDCD4/ PI3K/AKT/GSK-3 $\beta$ pathway in SH-SY5Y cells. Biomed Pharmacother. 2018;101:1003-1007.

37. Ghaderi S, Alidadiani N, Dilaver N, Heidari HR, Parvizi R, Rahbarghazi R, Soleimani-Rad J, Baradaran B. Role of glycogen synthase kinase following myocardial infarction and ischemia-reperfusion. Apoptosis. 2017;22:887-897.

38. Miura T, Tanno M. Mitochondria and GSK-3beta in cardioprotection against ischemia/reperfusion injury. Cardiovasc Drugs Ther. 2010;24:255-263.

39. Chen X, Yan X, Guo L. Inhibitory effect of Patrinia on BRL-3A cell apoptosis through the TLR4/PI3K/AKT/GSK3 $\beta$ and TLR4/P38/ JNK signaling pathways. Mol Med Rep. 2018;17:5344-5349.

40. Wang D, Zhang X, Li D, Hao W, Meng F, Wang B, Han J, Zheng Q. Kaempferide protects against myocardial ischemia/reperfusion injury through activation of the PI3K/Akt/GSK-3 $\beta$ pathway. Mediators Inflamm. 2017;2017:5278218.

41. Wu N, Zhang X, Bao Y, Yu H, Jia D, Ma C. Down-regulation of GAS5 ameliorates myocardial ischaemia/reperfusion injury via the miR-335/ROCK1/AKT/GSK-3 $\beta$ axis. J Cell Mol Med. 2019;23:84208431 . 